\title{
Association of Diabetes Mellitus and Histopathological Diagnosed Lichen Planus
}

\author{
Dimple Darad and Savitri Chauhan* \\ Department of Pathology, GMERS Medical College, Gotri, Vadodara Gujarat, India
}

\section{ABSTRACT}

Background: It is reported, that the presence of lichen planus among diabetic patients and the presence of diabetes in patients who are suffering from oral lichen planus has a correlation. Diabetes is being treated with antidiabetic drugs and certain antidiabetic drugs can cause an allergic manifestation to produce lichenoid reaction. Hence the present aim of study was to find out the prevalence of lichen planus among diabetic patient.

Materials \& Methods: Present study was carried out at a department of pathology GMERS Medical College, Gotri, Vadodara, Gujarat. All the participants did underwent the history, general and systemic examination which was followed by biochemical investigation for the blood sugar that is estimation of fasting blood sugar. Detailed history with clinical and dermatological examination was done. Fasting blood sugar was analysed. Skin biopsy was being taken from the skin lesion site and its histopathological examination was being done.

Results: On the basis of the biochemical analysis for diabetes, out of 100 patients included in the study, 49 were confirmed with the presence of diabetes. All the patients confirmed with diabetes were in type II diabetic patients, none of them were diagnosed with type I diabetes. There was significant relation between the presence of lichen planus and type II diabetes with $\mathrm{p}$ valus $<0.05$.

Conclusion: Lichen planus is a disease of adults (20-40 years) according to western data (Andreason, 1968; Scully, 1985) but in Indian population it is also common in the paediatric age group. The excess sugar in diabetes may not be a cause for lichen planus as nondiabetic patients also develops lichen planus, anxiety and stress seems to be associated with diabetes and this may be a reason precipitating lichen planus in diabetic patients.

Keywords: Diabetes Mellitus, Lichen Planus, Correlation, Histopathology

\section{Introduction}

Lichen Planus (LP) occurred followed by an inflammatory reaction to skin tissue with unknown etiology. Lichen planus although recognized over hundred years from now, its etiopathogenesis has not been understood in depth and conclusively. ${ }^{[I]}$ Over these years, many researchers have worked to determine the etiological factors, pathogenesis, characteristics and management of lichen planus. Lichen planus is a relatively common mucocutaneous disorder that affects approximately $0.1 \%$ to $2.0 \%$ of the general western population. 22$]$

Although the pathogenesis is not exactly known, but autoimmune reaction is the most probable reason. This is theorized that when an antigen such as virus or drug enters to a prone body and could transfer to epidermal keratinocytes and induces antigen changes in these cells. Therefore, the keratinocytes will be rejected by the immune system. Defects in expressing carbohydrates in epidermal cells, and abnormal enzyme activities that may induce disabled hormones for metabolic processes are other causes of LP. ${ }^{[3,4]}$
Cutaneous lichen planus is characterized by polygonal flattopped, violaceous papules and plaques, which in some cases can be intensely itchy. The lesions may result in longstanding residual hyperpigmentation, especially in dark skinned patients. LP has characteristic histopathological features which make the diagnosis relatively easy. The oral lesions are frequently found on the buccal mucosa, tongue, soft palate, gingival, and lips and they may have a variety of clinical appearances. ${ }^{[5]}$

Diabetes mellitus is recognized as a group of metabolic disorders characterized by chronic hyperglycemia and glucose intolerance, due to insulin deficiency, impaired effectiveness of insulin action or both. ${ }^{[6]}$ Among other manifestations, oral lichen planus have been reported to occur in diabetic patients and is due to prolonged state of immune suppression and acute hyperglycemia which causes alteration in immune responsiveness in diabetic patients. Hepatitis $\mathrm{C}$ and diabetes mellitus are major public health concern globally. ${ }^{[7]}$

Most of the associations between lichen planus and systemic diseases remain controversial as they rely on 
isolated, anecdotal evidence. It has been proposed that the endocrine dysfunction in diabetes mellitus may be related to immunologic defect that may also contribute to the development of Lichen planus. ${ }^{[8]}$

It is reported, that the presence of lichen planus among diabetic patients and the presence of diabetes in patients who are suffering from oral lichen planus has a correlation. Diabetes is being treated with antidiabetic drugs and certain antidiabetic drugs can cause an allergic manifestation to produce lichenoid reaction. Hence the present aim of study was to find out the prevalence of lichen planus among diabetic patient.

\section{Materials \& Methods}

Present study was carried out at a department of pathology GMERS Medical College, Gotri, Vadodara, Gujarat. for the period of one year. The study group included all the individuals, who were clinically diagnosed with lichen planus and latter on the diagnosis was confirmed by histopathology also. All the individuals were informed about the study and were informed about the whole procedure. Written informed consent was obtained from all of them. The ethical clearance certificate was obtained from the ethical committee of the institute.

All the participants did underwent the history, general and systemic examination which was followed by biochemical investigation for the blood sugar that is estimation of fasting blood sugar. Detailed history with clinical and dermatological examination was done. Fasting blood sugar was analysed. Minimization of the bias was done with careful history recording, clinical examination, histopathological evaluation andn biochemical investigation.

Inclusion \& Exclusion criteria were as follows: the patient of 18 or above 18 years, either of sex, presence of skin/ oral lichen planus, histopathological confirmed diagnosis, willingness to qparticipate in the research were included in the study. Patients aged below 18years, patients receiving treatment ( 6 months or more) for lichen planus such as systemic corticosteroids, retinoid or methotrexate, pregnant and lactating women, patients with lichenoid drug eruption, and the patients not willing to participate in the study were excluded from the study.

For statistical analysis we entered the data from the check lists to SPSS version 15. For describing continues data, we used the mean \pm standard deviation (SD). Frequency and percentage describe categorical variables. We used Chi2 test to compare our findings with predicted prevalence which was announced by previous studies. A p-value less than 0.05 were considered significant.

\section{Results}

On the basis of the inclusion and exclusion criteria a total of 100 patients were included in the study. All the 100 patients were histopathological confirmed with the diagnosis of oral lichen planus. Out of the total 100 patients, 67 were females and 33 were males. The mean age among all the patients were found to be 48 years. We used the criteria employed by American Diabetes Society in 2007 to confirm the presence of diabetes.

On the basis of the biochemical analysis for diabetes, out of 100 patients included in the study, 49 were confirmed with the presence of diabetes. All the patients confirmed with diabetes were in type II diabetic patients, none of them were diagnosed with type I diabetes. There was significant relation between the presence of lichen planus and type II diabetes with $p$ valus $<0.05$. (Table 2 )

The mean age of the LP patients with DM was significantly more than those who were not affected by DM, (56 years comparing to 48 years). The sex distribution was not significantly different for both groups. Among all patients $32(40 \%)$ were presented only with skin lesions, $6(7.5 \%)$ had only oral lesions and $44(52.4 \%)$ had both skin and oral cavity lesions. There was no significant relationship between the pattern of the distribution of lichenoid lesions and the presence of DM.

Table 1: Demographic characteristic of the patients.

\begin{tabular}{|c|c|c|c|}
\hline Age range & No. of Patients & Males & Females \\
\hline $18-30$ & 14 & 5 & 9 \\
\hline $31-40$ & 21 & 8 & 13 \\
\hline $41-50$ & 18 & 4 & 8 \\
\hline $51-60$ & 17 & 9 & 9 \\
\hline $61-70$ & 11 & 2 & 8 \\
\hline $71-80$ & 11 & 3 & 6 \\
\hline $81-90$ & 8 & 2 & 37 \\
\hline
\end{tabular}


Table 2: Correlation between lichen planus and presence of diabetes.

\begin{tabular}{|c|c|c|c|}
\hline FBS $(\mathbf{m g} / \mathbf{d l})$ & Cases & Percent & P - Value \\
\hline$>100$ & 49 & 49 & \multirow{2}{*}{0.03} \\
\hline$<100$ & 51 & 51 & \\
\hline Total & 100 & 100 & \\
\hline
\end{tabular}

Table 3: Patients with clinical evidence of oral lichen planus.

\begin{tabular}{|c|c|c|}
\hline Lesions & Diabetic Patients $(\mathbf{n}=\mathbf{4 9})$ & Non Diabetic Patients $(\mathbf{n}=\mathbf{5 1})$ \\
\hline Reticular & 23 & 21 \\
\hline Atrophic & 11 & 5 \\
\hline Hyperplastic & 8 & 0 \\
\hline Erosive & 7 & $\mathbf{5 1}$ \\
\hline Total & $\mathbf{4 9}$ & 25 \\
\hline
\end{tabular}

\section{Discussion}

LP is defined as an inflammatory keratotic dermatitis with an unknown origin $(2,3,12)$. Although the studies about the relationship between DM and LP have been progressed during the last decades, the results are in conflict. Lichen planus was seen at various sites in oral cavity. ${ }^{[9]}$ Shklar and McCarthy found $80 \%$ of involvement of buccal mucosa and Shklavouhou reported about $83 \%$ involvement of buccal mucosa with buccal mucosa showing higher predominance. ${ }^{[10]}$

Lichen planus is a common complex inflammatory disease that shares many immunological features with other common complex disorders such as cardiovascular disease, obesity, diabetes, depression and dyslipidemia. [11] The diseases that occur concurrently are often thought to be related to common pathogenetic mechanisms. Comorbidities are most likely related to underlying disease pathogenesis and exclude factors such as lifestyle, access to healthcare and patients' associated economic status.

In our series, maximum numbers of patients were seen in the age group of 20-40 years. This correlates with other studies that describes data of Indian population (Bhattacharya et al. ${ }^{[12]}, 2000$; Singh and Kanwar ${ }^{[13]}$, (1976).

Patients with lesions who were taken medications that have been associated with mucosal reactions were informed of the possible implication of their medications. However, the medication implicated with the lesions prescribed by a physician other than the physician treating the diabetes. Despite the patients who were taken these medications showed any evidence of lichen planus or lichenoid lesions. The types of medications that were associated with the oral lesions, NSAIDs (non steroidal anti inflammatory drugs) and ACE (angiotensin converting enzyme) inhibitors, were statistically significant $(\mathrm{p}<0.005)$ which is similar to the findings of Robertson and Wray. ${ }^{[8,14]}$
In last decade, in 2000 , Guggenheimer, et al ${ }^{[15]}$ reported the incidence rate of $0.5 \%$ of oral LP among patients with insulin dependent DM. In 2004, the prevalence of DM in 140 Turkish people with LP was revealed as $15.7 \%$. Moreover, in 2007 , the prevalence of DM in patients with LP was found as $26.7 \%$ in that country. In addition, they found a significant difference between the concentration of $\mathrm{HbA} 1 \mathrm{C}$, fasting blood sugar and insulin resistance in patients with LP and the control group. In the present study there are $49(49 \%)$ cases who had FBS $>100$ which is statistically significant $(\mathrm{P}=0.03)$. This finding corroborates with other studies. Several epidemiological surveys have been conducted on association of Diabetes with Lichen planus.

Conclusion:Lichen planus is a disease of adults according to western data but in Indian population it is also common in the paediatric age group. The excess sugar in diabetes may not be a cause for lichen planus as nondiabetic patients also develops lichen planus, anxiety and stress seems to be associated with diabetes and this may be a reason precipitating lichen planus in diabetic patients.

\section{References}

1. Carrozzo M, Thorpe RJ: Update on oral lichen planus. Expert Review of Dermatology 2009;4:483-94.

2. Huber MA: Oral lichen planus. Quintessence international 2004, 35 .

3. Atefi N, Majedi M, Peyghambari S, Ghourchian S: Prevalence of diabetes mellitus and impaired fasting blood glucose in patients with Lichen Planus. Medical journal of the Islamic Republic of Iran 2012;26:22.

4. Herling A, König M, Bulik S, Holzhütter HG: Enzymatic features of the glucose metabolism in tumor cells. The FEBS journal 2011;278:24:36-59.

5. Eisen D: The clinical features, malignant potential, and systemic associations of oral lichen planus: a study of 723 
patients. Journal of the American Academy of Dermatology 2002;46:207-14.

6. Gavin III JR, Alberti K, Davidson MB, DeFronzo RA: Report of the expert committee on the diagnosis and classification of diabetes mellitus. Diabetes care 1997;20:1183.

7. SHAH SMA, KUNDI JA, KHATTAK MSK, Ilyas M, Gul F, Khan S: Association of oral lichen planus with hepatitis-C and diabetes mellitus. Pakistan Oral \& Dental Journal 2016:36.

8. Van Dis ML, Parks ET: Prevalence of oral lichen planus in patients with diabetes mellitus. Oral Surgery, Oral Medicine, Oral Pathology, Oral Radiology, and Endodontology 1995;79:696-700.

9. Alrashdan MS, Cirillo N, McCullough M: Oral lichen planus: a literature review and update. Archives of dermatological research 2016;308:539-51.

10. McCarthy PL, Shklar G: Diseases of the oral mucosa: Lea \& Febiger, 1980.
11. Varma K, Shukla P: Association of diabetes mellitus in patients with Lichen Planus. Indian Journal of Clinical and Experimental Dermatology 2017;3:14-6.

12. Bhattacharya M, Kaur I, Kumar B: Lichen planus: a clinical and epidemiological study. The Journal of dermatology 2000;27:576-82.

13. Singh O, Kanwar A: Lichen planus in India: an appraisal of 441 cases. International journal of dermatology 1976;15:752-6.

14. Robertson WD, Wray D: Ingestion of medication among patients with oral keratoses including lichen planus. Oral surgery, oral medicine, oral pathology 1992;74:183-5.

15. Guggenheimer J, Moore PA, Rossie K, Myers D, Mongelluzzo MB, Block HM, Weyant R, Orchard T: Insulindependent diabetes mellitus and oral soft tissue pathologies. II. Prevalence and characteristics of Candida and candidal lesions. Oral Surgery, Oral Medicine, Oral Pathology, Oral Radiology and Endodontics 2000;89:570-6.

*Corresponding author:

Dr Savitri Chauhan, MD, Associate Professor, Department of Pathology, GMERS Medical College, Gotri, Vadodara Gujarat(India)- 390021

Phone: +91 9898067478

Email: savitri_chauhan@yahoo.com

Financial or other Competing Interests: None. 